\title{
Cloud Computing Resource Scheduling Research Based on the Improved Quantum Genetic Algorithm
}

\author{
Lijun Mao, Xinyan Wang, Jing Li \\ School of Intelligence Science and Information Engineering \\ Xi'an Peihua University, Xi'an, China \\ 35909250@qq.com, 36404843@qq.com, 28328715@qq.com
}

Keywords: Cloud computing; Kvalue clustering; Genetic algorithm

\begin{abstract}
By aiming at the situation of applying the existing intelligent algorithm to the cloud computing job scheduling and giving analysis and comparison, it proposes the method combining $K$ value clustering with genetic algorithm. The improved genetic algorithm with the solved individual fitness in genetic algorithm corresponding to the resource sequence in cloud computing and obtained after dynamic adjustment of rotation angle and quantum mutation and crossover has global searching ability, and can then avoid disadvantages of the existing algorithm effectively and realize optimized solution of the problems finally.
\end{abstract}

\section{Introduction}

Along with rapid development of information technology and internet technology, data size on Web shows rapid growth with unprecedented speed[1]. For uneven distribution of network resource and declination of effective utilization, the problem about handling capacity of internet to the mass data is prominent day by day, and it becomes the research focus of internet community to share network resource maximized. Cloud computing is a new business computing module emerged in internet community, business community and the general background of individual demand. Currently, many IT companies, universities and research institutions consider cloud computing as strategic target and scientific research hotspot[2].

Considering heterogeneity of infrastructure, the reasonable resource scheduling method becomes the key affecting cloud service quality[3]. By aiming at the solution to resource scheduling problem, it has the solution technology based on classic optimized problem, namely: summarizing the scheduling problem to some known mathematical problem, creating model on this basis, and adopting classic solution method of this mathematical problem, taking the cloud computing network planning model based on demand and supply equilibrium mechanism and the method of distributing cloud computing resource by introducing cuckoo intelligent algorithm as example, whose research results can promote the distribution efficiency in cloud computing environment effectively.

\section{Cloud Computing Resource Model}

\subsection{Scheduling model}

One of the key technology of cloud computing system is server virtualization technology, which can realize separation of service layer and physical resource layer of cloud computing[4], assign workload to different physical resources flexibly and realize efficient use of resource.

Supposing that there are $\mathrm{n}$ tasks on different cloud nodes in cloud computing $T=\left\{T_{1}, T_{2}, \ldots T n\right\}$ and the limited resource nodes $G=\left\{G_{1}, G_{2}, \ldots G_{n}\right\}$, task $T_{i}$ shall be completed on resource $G_{j}$, optimal solution of individual fitness shall be the best resource allocation sequence when corresponding each 
individual in genetic algorithm to resource sequence, and expression of the function relationship that $i$ subtask corresponds to resource $j$ shall be as follows[5]:

$$
F\left(y_{i j}\right)=C(i, j)+E(i, j)+T(i, j)
$$

The resource allocation result in cloud computing and obtained from this formula is the minimum value of resource consumed after completing all subtasks, $E(i, j)$ namely $\min \sum_{k=1}^{N} F_{k}\left(y_{i j}\right)$, of which $C(i, j)$ refers to consumption cost of subtask $i$ on resource $j$, refers to energy consumption of task $i$ on resource $j$, and $T(i, j)$ refers to time consumption of task $i$ on resource $j$.

\subsection{Problems existed in the current scheduling algorithm}

In cloud computing environment, the number of users needing service is huge, and work quantity and data size submitted by user are also great. Thus, to try the best to satisfy each user when handling multi-user scheduling problem and serving more users, it needs to consider not only the shortest completion time of all works submitted by all users but also response time of each user, so that the user may not need to wait for a too long time period[6]. Some of the existing scheduling algorithms consider total completion time of the works merely while does not consider average completion time of each user, which may result in loss of some potential fine genes, for total completion time of the works of all users shall be lesser hen average work completion time of each user is lesser[7]. Thus, this paper determines to combine quantum genetic algorithm with $K$ value clustering algorithm to avoid local optimum.

\section{Basic Algorithm Description}

\subsection{Quantum Genetic Algorithm}

Quantum genetic algorithm is probability optimization method based on quantum computing principle, which applies probability amplitude expression of quantum bit to chromosomal coding and makes one chromosome possessing superposition of several modes, so it has more parallelism by comparing with the traditional algorithm[8]. In quantum genetic algorithm, chromosomal coding is not expressed by determined value while is expressed by quantum bit.

In this way, it makes chromosome expressing several states, and can reduce group scale, increase group diversity and avoid the precocity occurred in genetic algorithm.

\section{(1) Quantum Bit Coding}

The medium acting as information memory cell in quantum computing is quantum bit. In algorithm, quantum bit is expressed by arbitrary value in the two states, and it can express random infinite intermediate states between 0 and 1 in binary system, as follows:

$$
|\varphi\rangle=\alpha|0\rangle+\beta|1\rangle
$$

Of which, $\alpha$ and $\beta$ are plural relationship, and $|\alpha|^{2}$ and $|\beta|^{2}$ refer to $|0\rangle$ and $|1\rangle$ states of quantum respectively, which shall satisfy the following relationship:

$$
|\alpha|^{2}+|\beta|^{2}=1
$$

Thus, the system possessing $m$ quantum bits can be described as follows:

$$
\left|\begin{array}{c|c|c|c|}
\alpha_{1} & \alpha_{2} & \cdots & \alpha_{m} \\
\beta_{1} & \beta_{2} & \cdots & \beta_{m}
\end{array}\right|
$$


When $m$ quantum bits can contain $2^{m}$ state information simultaneously, it can increase group diversity and ensure the stronger global searching ability.

In this paper, evolution rule of quantum genetic algorithm is realized by quantum revolution door which is defined as follows :

$$
\begin{gathered}
\mathrm{U}(\theta)=\left(\begin{array}{cc}
\cos \theta & -\sin \theta \\
\sin \theta & \cos \theta
\end{array}\right) \\
\left(\begin{array}{c}
\alpha_{\mathrm{i}}^{\mathrm{t}+1} \\
\beta_{\mathrm{i}}^{\mathrm{t}+1}
\end{array}\right)=\mathbf{U}(\theta)\left(\begin{array}{l}
\alpha_{\mathrm{i}}^{\mathrm{t}} \\
\beta_{\mathrm{i}}^{\mathrm{t}}
\end{array}\right)=\left(\begin{array}{cc}
\cos \theta & -\sin \theta \\
\sin \theta & \cos \theta
\end{array}\right)\left(\begin{array}{l}
\alpha_{i}^{t} \\
\beta_{i}^{t}
\end{array}\right)
\end{gathered}
$$

Of which, $\left[\alpha_{i}^{t} \beta_{i}^{t}\right] \mathrm{T}$ refers to the $\mathrm{i}$ quantum bit of $\mathrm{t}$ generation of quantum chromosome, and $\left[\alpha_{\mathrm{i}}^{\mathrm{t}+1} \beta_{\mathrm{i}}^{\mathrm{t}+1}\right] \mathrm{T}$ refers to the $\mathrm{i}$ quantum bit of $\mathrm{t}+1$ generation of quantum chromosome after mutation.

(2) Insufficiencies of the Algorithm Analysis

The improper adjustment strategy of quantum genetic algorithm may be easy to cause convergence and precocity of the algorithm, which may result in the situation that chromosomal gene position should not be close to the contemporary optimal quantum chromosomal gene position and the existing quantum chromosome should deviate from the current optimal chromosome thereof, so the performance of revolution door is crucial to the promotion of algorithm performance.

\section{2 $\mathrm{K}$ value Clustering Algorithm}

\section{(1) Algorithm Description}

$K$ value clustering algorithm is an algorithm based on partition clustering algorithm, with main process as follows: setting value of the number $K$ of partition clusters, and then selecting $K$ initial data objects randomly as cluster center of the initial solution of the algorithm, calculating distance from each data object to the $K$ cluster centers, partitioning data object to the cluster of the nearest center, adjusting new cluster, computing center of the new cluster again, and finishing the algorithm when the two cluster centers calculated should show no change[9]. $K$ value clustering algorithm process shall show continuous iteration, it needs to inspect correctness of the sample data classification in each iteration process, and corresponding adjustment shall be needed for incorrectness.

Basic steps of the traditional value clustering algorithm are as follows[10]:

a. Select k points as initial mass center.

b. Repeat the following process: assign each point to the nearest mass center, form k clusters, and calculate mass center of each cluster again.

c. Finish the algorithm when mass center should show no change.

This algorithm has no high requirements to the number of initial clusters and the selection of initial mass center, while it can reduce operation time of the algorithm and promote efficiency by selecting number of initial clusters and initial mass center reasonably.

Selection of initial mass center can adopt the method based on density to further promote clustering efficiency. However, to show the effectiveness of the dynamic clustering algorithm which determines $K$ value based on multi-way tree, the method of selecting initial mass center randomly and uniformly shall be adopted.

(2) Insufficiencies of the Algorithm Analysis

$K$ value clustering algorithm possesses such advantages as rapid and simple computing and lesser computing steps, and it has the problems as follows:

a. When conducting data clustering, user cannot know the proper number of clusters, namely definition of $\mathrm{K}$ value;

b. Selection of initial cluster center has great dependence, and it is easy to cause local optimum of 
the algorithm.

Thus, it is urgent to improve the two algorithms to solve the problem of low performance caused by single algorithm.

\subsection{Combination and Improvement of the Two Algorithms}

To adapt to the cloud computing resource scheduling model in this paper, we give corresponding improvements to quantum genetic algorithm and $\mathrm{K}$ value clustering algorithm and realize effective fusion of the two algorithms improved.

\section{(1) Improved $K$ value Clustering Algorithm}

By aiming at the problem that initial cluster center is easy to fall into local optimum in $\mathrm{K}$ value clustering algorithm, this paper proposes the algorithm with rotary improvement of the coordinates of cluster center:

Definition 1: supposing that the Euclidean distance between the two $N$-dimensional vectors

$$
\begin{array}{r}
x_{i}=\left(x_{i 1}, x_{i 2}, \cdots x_{i N}\right)^{T} \text { and } \quad x_{j}=\left(x_{j 1}, x_{i 2}, \cdots x_{j N}\right)^{T} \text { is: } \\
d\left(x_{i}, x_{j}\right)=\left|x_{i}-x_{j}\right|=\sqrt{\sum_{k=1}^{N}\left|x_{i k}-x_{j k}\right|}
\end{array}
$$

Definition 2: supposing the two $N$-dimensional data sample points as $X=\left(x_{1}, x_{2}, \cdots x_{n}\right)$ and $Y=\left(y_{1}, y_{2}, \cdots y_{n}\right)$ and coordinates of the center point as:

$$
\operatorname{Mid}(x, y)=\left(\frac{x_{1}+y_{1}}{n}, \frac{x_{2}+y_{2}}{n}, \cdots, \frac{x_{n}+y_{n}}{n}\right)
$$

\section{(2) Improvement of Quantum Genetic Algorithm}

We can update the group by adjusting rotation angle of quantum door dynamically, and rotation angle $\theta$ is defined as:

$$
\theta=S\left(\alpha_{i}, \beta_{i}\right) \Delta \theta_{i}
$$

In the formula, $S\left(\alpha_{i}, \beta_{i}\right)$ refers to rotation direction, and $\Delta \theta_{i}$ refers to step length. When setting value of $\Delta \theta_{i}$ as 0 or $d e l$, we can better adjust quantum selection mechanism.

$$
\operatorname{del}=0.04 \pi\left(1-k \cdot \frac{n}{\operatorname{Max}+1}\right)
$$

In the formula, value of $k$ is a constant among $(0,1), n$ and Max refer to the current number of iterations and the maximum number of iterations, which can ensure that $\mathrm{del}$ approaches to $0.04 \pi$ in the initial stage of algorithm and $0.04 \pi(1-k)$ in the later stage of algorithm.

\section{(3) Quantum Mutation and Crossover}

We can realize update of group by improving rotation angle, and it needs to introduce mutation and crossover to avoid local optimum. The core of genetic algorithm is crossover operation, new individual can be generated in the crossover operation, and adaptation of the new individual may be enhanced for gene recombination, which could then promote development of the group towards better direction. When giving crossover computing, the group information shall show full combination and exchange, efficiency shall be promoted, and quantum crossover shall be executed. 
Excepting for the optimal individuals in the group, some individuals can be selected according to certain crossover probability, as shown in Fig. 1:

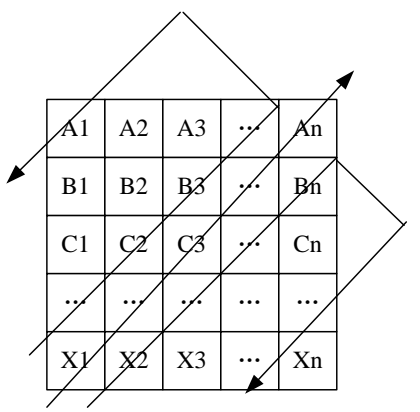

Figure 1. Quantum Crossover and Mutation

\section{Description of the Simulation Experiment}

This paper gives analysis to the back-end data of cloud video website platform based on J2EE framework, and corresponding parameters of genetic algorithm and $K$ value clustering algorithm are set on Matlab 2012 platform.

When supposing number of virtual tasks in cloud computing as 200 and number of virtual resources as 10, network consumption cost rate and network consumption time are as shown in Fig. 2-3. It can be seen from the Fig. that the two indicators of this algorithm show stable growth along with gradually increment of the number of tasks, which indicates that this algorithm is more stale and can dispose task faster, reduce cost consumption and shorten operation time.

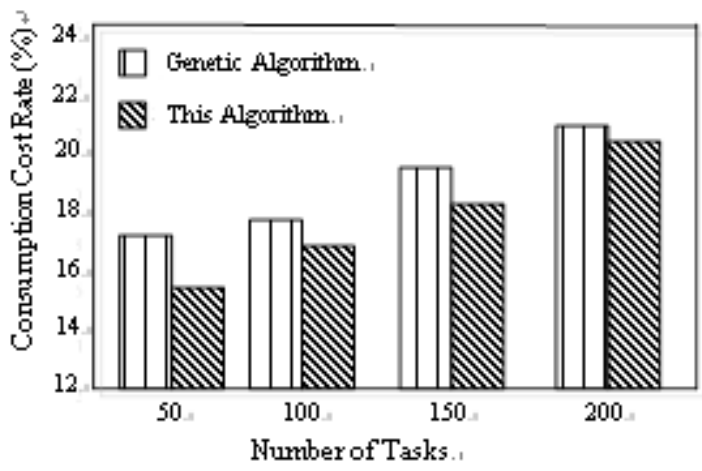

Figure 2. Comparison of Two Tasks' Consumption Cost in Two Algorithms

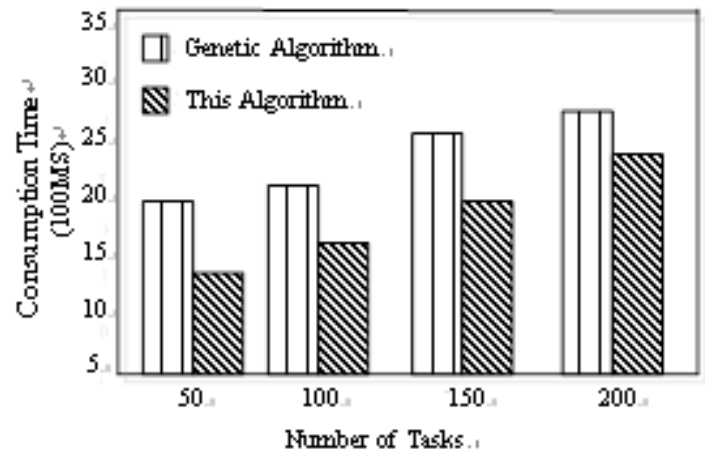

Figure 3. Comparison of Two Tasks' Time Consumption in Two Algorithms 


\section{Conclusions}

This paper introduces $K$ value clustering algorithm and genetic algorithm by aiming at the resource scheduling problem of cloud computing, gives improvement by aiming at insufficiencies of the two algorithm, realizes effective fusion of the two improved algorithms, and conducts simulation experiment in Matlab platform. The results indicate that this algorithm can promote resource allocation efficiency of cloud computing effectively and reduce payment cost of cloud service provider obviously.

\section{Acknowledgment}

This work was supported by Shaanxi Province Education Science Planning Topic "Empirical Research on Teaching Reform of the Course Fundamentals of Computer Application Based on Working Process Systematization in the Background of Transformation and Development" under Grant No. SGH17H461. It is also supported by the Natural Science Foundation of Xi'an Peihua university "Research on the Application of an Improved Quantum Genetic Algorithm in Resource Allocation under Cloud Computing” under Grant No.PHKT17015.

\section{References:}

[1] Feng Liu, Li Bi and Jun Yang. 2016.An Improved Genetic Algorithm Used in Cloud Computing Resource Scheduling [J]. Computer Measurement and Control, 24(5):202-205

[2]Wenzhong $\mathrm{Xu}$, Zhiping Peng and Jinglong Zuo. 2015.Cloud Computing Resource Scheduling Strategy Research Based on Genetic Algorithm [J]. Computer Measurement and Control, 23(5):1653-1655

[3] Xianglai Meng and Xiaoyu Ma. 2015. Optimal Scheduling Model Simulation of Network Resource Matching in Cloud Computing [J]. Bulletin of Science and Technology, 31(7):186-189

[4] Hongwei Zhao and Shengpu Li. 2016.Research on the Cloud Computing Resource Scheduling Method Based on Particle Swarm Algorithm and RBP Neural Network [J]. Computer Science, 43(3):113-117

[5] Shunping Wang and Jie Wang. 2015.Multisource Resource Access Characteristics of Heuristic Cloud Computing - The Minimum Variance Estimation [J]. Bulletin of Science and Technology, 31(4):133-135

[6] Huaqiao Ye and Shanting Ding. 2014.Research on the Improved Cuckoo Algorithm in Cloud Computing Resource [J], Computer Measurement and Control, 22(12):4150

[7] Shiyang Ye, Wenbo Zhang and Hua Zhong. 2015.Virtual Resource Scheduling Method Towards SLA in Cloud Computing Environment [J], Computer Application and Software, 32(4):11-17

[8] Lijuan Zhou and Chunying Wang. 2015.Cloud Computing Resource Scheduling Strategy Research Based on Particle Swarm Optimization Algorithm [J]. Computer Science, 42(6):279-281

[9] Minda Hu. 2017. Research on the Fair Allocation Strategy with Efficient Resource Use in Cloud Computing Environment. [D]. Nanjing University of Information Science \& Technology.

[10] Ke Li. 2017.Research on the Resource Allocation Based on Ant Colony Optimization Algorithm in Cloud Computing. [D]. Hangzhou Dianzi University. 